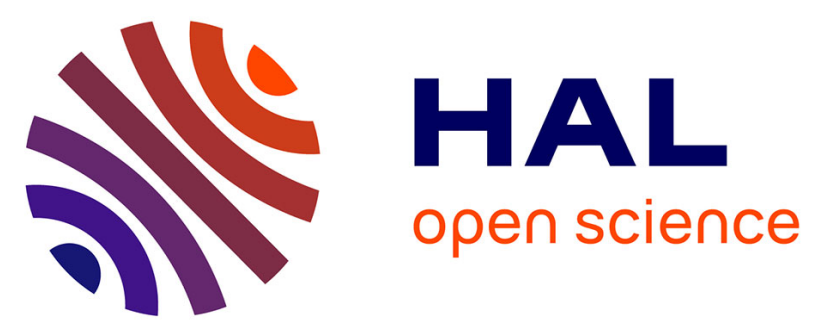

\title{
Exposure of children to metals via tap water ingestion at home: Contamination and exposure data from a nationwide survey in France
}

Barbara Le Bot, Jean-Paul Lucas, Francoise Lacroix, Philippe Glorennec

\section{To cite this version:}

Barbara Le Bot, Jean-Paul Lucas, Francoise Lacroix, Philippe Glorennec. Exposure of children to metals via tap water ingestion at home: Contamination and exposure data from a nationwide survey in France. Environment International, 2016, 94, pp.500-507. 10.1016/j.envint.2016.06.009 . hal01390976

HAL Id: hal-01390976

https://hal-univ-rennes1.archives-ouvertes.fr/hal-01390976

Submitted on 23 Feb 2017

HAL is a multi-disciplinary open access archive for the deposit and dissemination of scientific research documents, whether they are published or not. The documents may come from teaching and research institutions in France or abroad, or from public or private research centers.
L'archive ouverte pluridisciplinaire HAL, est destinée au dépôt et à la diffusion de documents scientifiques de niveau recherche, publiés ou non, émanant des établissements d'enseignement et de recherche français ou étrangers, des laboratoires publics ou privés. 
The exposure of children to metals via ingestion of tap water at home: contamination and exposure data from a nationwide survey in France.

Barbara le Bot ${ }^{1,2^{\star}}$, Jean-Paul Lucas ${ }^{3,4}$, Françoise Lacroix ${ }^{1,2}$, Philippe Glorennec ${ }^{1,2}$

${ }^{1}$ EHESP, School of Public Health, Rennes, Sorbonne Paris Cité - Avenue du Professeur LéonBernard, CS 74312, 35043 Rennes cedex, France.

${ }^{2}$ Irset Inserm, UMR 1085-Institut de Recherche sur la Santé, l'Environnement et le Travail, Rennes, France.

${ }^{3}$ Paris Est University - CSTB - Scientific and Technical Building Centre, Marne la Vallée cedex 2, France

${ }^{4}$ University of South Brittany, UMR 6205, LMBA, F-56000 Vannes, France

\section{$\left(^{*}\right)$ Corresponding author.}

E-mail address: barbara.lebot@ehesp.fr (Barbara Le Bot)

Phone: +332 99022924

Fax: +33299022929 


\begin{abstract}
29 inorganic compounds (Al, As, B, Ba, Be, Bi, Ca, Cd, Ce, Co, Cr, Cu, Fe, Gd, K, Mg, Mn, Mo, $\mathrm{Na}, \mathrm{Nd}, \mathrm{Ni}, \mathrm{Pb}, \mathrm{Sb}, \mathrm{Se}, \mathrm{Sr}, \mathrm{TI}, \mathrm{U}, \mathrm{V}$ and $\mathrm{Zn}$ ) were measured in the tap water of 484 representative homes of children aged 6 months to 6 years in metropolitan France in 2008-2009. Parents were asked if their children consume tap water. Sampling design and sampling weights were taken into account to estimate element concentrations in tap water supplied to the $3,581,991$ homes of 4,923,058 children aged 6 months to 6 years. Median and 95th percentiles of concentrations in tap water were in $\mu \mathrm{g} / \mathrm{L}: \mathrm{Al}:<10,48.3$, As: 0.2, 2.1; $\mathrm{B}:<100,100$; Ba : 30.7, 149.4; Ca: 85 000,121,700; Cd:<0.5, <0.5;Ce: <0.5, <0.5; Co:<0.5, 0.8; Cr : <5,<5 ; Cu : 70,720; $\mathrm{K}: 2210,6740$; Fe:<20, 46; Mn: <5, <5; Mo: <0.5, 1.5; Na: 14500, 66800; Ni : <2,10.2;Mg: 6500, 21200; $\mathrm{Pb}:<1,5.4$; Sb: <0.5, <0.5; Se: <1,6.7; Sr: 256.9, 1004; Tl: <0.5,<0.5; U: <0.5, $2.4 ; \mathrm{V}:<1,1 ; \mathrm{Zn}: 53,208$. Of the $2,977,123$ young children drinking tap water in France, some were drinking water having concentrations above the 2011 World Health Organization drinking-water quality guidelines: respectively 498 (Cl95\%:0-1,484) over $700 \mu \mathrm{g} / \mathrm{L}$ of Ba; 121,581 (Cl95\%:7091236,070) over $50 \mathrm{mg} / \mathrm{L}$ of $\mathrm{Na} ; 2044$ (Cl95\%: 0-6,132) over $70 \mu \mathrm{g} / \mathrm{L}$ of $\mathrm{Ni}$, and 78,466 (17,171$139,761)$ over $10 \mu \mathrm{g} / \mathrm{L}$ of $\mathrm{Pb}$. Being representative, this tap water contamination data can be used for integrated exposure assessment, in conjunction with diet and environmental (dust and soil) exposure data.
\end{abstract}

Keywords: inorganic, drinking-water, metal, metalloid, environmental exposure 


\section{Funding}

This research was funded by the French ministries in charge of health, ecology, and housing. Funding bodies did not take part in study design, data analysis nor interpretation. 


\section{Introduction}

Water is sourced for potabilization from various supplies: and its quality depends on water resource origin and possible contamination, as well as on subsequent water treatment processes such as filtration, aeration, and chemical precipitation, and by interaction with distribution system equipment (Dinelli et al., 2012). Drinking-water is essential to human health and french child consumers drink, on average, $250 \mathrm{ml}$ of tap water per day (Fantino and Gourmet, 2008), (Lioret et al., 2010). Tap water is a source of trace elements that are essential to life, such as $\mathrm{Ca}, \mathrm{Mg}, \mathrm{Fe}$, and $\mathrm{Zn}$ - yet could also be contaminated by heavy metals or metalloids such as $\mathrm{As}, \mathrm{Pb}, \mathrm{TI}$, or $\mathrm{U}$, which are recognized to be toxic (Villaescusa and Bollinger, 2008), (Zietz et al., 2010), (Peter and Viraraghavan, 2005). Heavy metals can be toxic at very low levels of concentration. Ingested metals can accumulate in the body, affecting the nervous system, especially children during the period of maximum brain growth, disrupting normal functioning of internal organs and acting as cofactors in other diseases. Recently, some heavy metals have been associated with infertility (Giaccio et al., 2012). Assessment of human exposure to heavy metals is increasingly being investigated in all environmental media - such as dust and soil (Ibanez et al., 2010) (Mielke et al., 2010), (Glorennec et al., 2012), and water (Dinelli et al., 2012), (Nahar and Zhang, 2012), (Stalder et al., 2012) in order to evaluate the impact of environmental pollution on public health, particularly in children. Twelve metals or metalloids are regularly quantified in water to verify compliance with the concentration limit imposed by the EU Drinking Water Directive (European Directive 1998/83EC, 1998). Assessing health risks linked to metal ingestion via water requires local contamination data (in addition to values for ingested quantities) with a water sampling strategy adapted to end-consumers' exposure.

The objective of this study is to determine the metals and others elements concentrations to which children are exposed through drinking tap water at home in France. 29 elements were measured (Al, As, B, Ba, Be, Bi, Ca, Cd, Ce, Co, Cr, Cu, Fe, Gd, K, Mg, Mn, Mo, Na, Nd, Ni, Pb, 
$\mathrm{Sb}, \mathrm{Se}, \mathrm{Sr}, \mathrm{TI}, \mathrm{U}, \mathrm{V}$ and $\mathrm{Zn}$ ) in a perspective of representative and integrative chronic exposure assessment.

\section{Material and methods}

\subsection{Study design}

Our dwellings sample was derived a national lead poisoning survey ( $n=3,623$ children) (Etchevers et al., 2014) with a sampling design allowing to provide population based estimates. The design of the initial lead poisoning survey was a two-stage sampling, stratified at the first stage: the primary sampling units were hospitals within which the children were the second sampling units. Detailed Inclusion procedures and representativeness have been described and discussed by Etchevers et al (Etchevers et al., 2014). A subsample of 484 children and corresponding housing units was then selected and investigated. Homes were main residences (as opposed to second homes) in metropolitan France inhabited by at least one child aged 6 months - 6 years located as described with (Fig.1). The design and sampling weights were taken into account in a design-based analysis (Lumley, 2010a) to finally get population-based estimates of metals or elements concentrations in the tap water of the whole population. A poststratification on age, location and type (single or multi- units) was performed on the sampling weights to increase the precision of estimators, as described in details by Lucas et al. (2014). Environmental investigations (measurements and questionnaire) were carried out at the 484 homes between October 2008 and August 2009. For statistical calculations, values below the Limit of Quantification (LOQ) were assigned to the value of LOQ/2. Statistical analyses were performed with the "survey" $R \circledast 2.9 .0$ software package (Lumley, 2010b). 




Fig. 1. Location of the 484 tap water samples, France 2008-2009.

An individual written report on the results was sent to each family. Prior authorisation (Authorisation No. 908326) was obtained from the French Data Protection Authority (Commission Nationale de l'Informatique et des Libertés). Although already published elsewhere, results concerning lead concentration in water (Lucas et al., 2012), are also partially reported here, to ensure consistency. 


\subsection{Tap water use for drinking water}

A questionnaire was used to collect information about child behaviour. Parents were notably asked whether or not their child consumed tap water.

\subsection{Tap water measurement}

\subsubsection{Drinking-water sampling}

Tap water was sampled in the kitchen at random times of day, from Monday to Friday. Tap was long flushed. After a controlled 30 min stagnation time, a $2 \mathrm{~L}$ sample was taken in a high density polyethylene (HDPE) bottle, homogenized by shaking, and $250 \mathrm{ml}$ was immediately transferred into another HDPE bottle containing 1\% HNO3 (65-70\%) (Agence Française de Normalisation, 2004).

\subsubsection{Analysis}

Toxic metals or metalloid with all analysable elements together with sufficient performance to be quantified were analysed in tap water using an inductively-coupled plasma mass spectrometer (ICP-MS Agilent Technologie 7500ce equipped with a quadrupole mass filter and an octopole reaction cell) by ICPMS. The elements were $\mathrm{Al}, \mathrm{As}, \mathrm{B}, \mathrm{Ba}, \mathrm{Be}, \mathrm{Bi}, \mathrm{Ca}, \mathrm{Cd}, \mathrm{Ce}, \mathrm{Co}, \mathrm{Cr}, \mathrm{Cu}, \mathrm{Fe}$, $\mathrm{Gd}, \mathrm{K}, \mathrm{Mg}, \mathrm{Mn}, \mathrm{Mo}, \mathrm{Na}, \mathrm{Nd}, \mathrm{Ni}, \mathrm{Pb}, \mathrm{Sb} \mathrm{Se}, \mathrm{Sr}, \mathrm{Tl}, \mathrm{U}, \mathrm{V}, \mathrm{Zn}$. The system is equipped with an autosampler (CETAC ASX-510), a micro nebulizer (microflow) and a Scott chamber. The plasma is energized by radio frequency $(27.12 \mathrm{MHz})$. ICP/MS settings were as follows: sample feed rate of $0.3 \mathrm{ml} / \mathrm{min}$, RF power of $1550 \mathrm{~W}$ and plasma gas flow rate of $15 \mathrm{l} / \mathrm{min}$. The makeup gas flow rate was $0.2 \mathrm{l} / \mathrm{min}$, and the carrier gas flow rate was $0.8 \mathrm{l} / \mathrm{min}$. The mass spectrometer interface included a nickel sampling cone and a nickel skimmer cone.

29 elements were analysed simultaneously. Total duration of data acquisition was 202 seconds per sample. The acquisition parameters for each element were isotopic mass in atomic mass 
unit (amu), gas mode and acquisition time u(s): Be (9, no-gas mode, 0.3); B (10, no-gas mode, 0.3); $\mathrm{Na}$ (23, He mode, 0.3); Mg (24 and 26, He mode, 0.3); Al (27, He mode, 0.9); K (39, He mode, 0.3); Ca (43 and 44, no-gas mode, 0.3); V (51, He mode, 0.3); Cr (52 and 53, He mode, 0.3); Fe (54, 56 and 57, He mode, 0.3); Mn (55, He mode , 0.3); Co (59, He mode, 0.3); Ni (60 and 62, He mode, 0.3); Cu (65, He mode, 0.3); Zn (66, He mode, 0.3); Zn (67 and 68, He mode, 0.9); As (75, He mode, 1.5); $\mathrm{Se}$ (78, $\mathrm{H}_{2}$ mode, 1.5); $\mathrm{Sr}$ (86 and 88 , no-gas mode, 0.3); Mo (95 and 97, no-gas mode, 0.3); Cd (111 and 114, no-gas mode, 0.9); Sb (121 and 123, no-gas mode, 0.3); Ba (135 and 137, no-gas mode, 0.3); Ce (140, no-gas mode, 0.3); Nd (143 and 146, no-gas mode, 0.3); Gd (157, no-gas mode, 0.3); TI (203 and 205, no-gas mode, 0.3); Pb (206, 207 and 208, no-gas mode, 0.3); $\mathrm{Bi}$ (209, no-gas mode, 0.9); U (238, no-gas mode; 0.3). In order to suppress inter- element interference, we used corrected equations: one for Fe quantification (the isotope 54 amu was corrected by isotope 52 amu of $\mathrm{Cr}$ ), one for $\mathrm{Cd}$ quantification (the isotope 114 amu was corrected by isotope 118 amu of Sn) and for In quantification (the isotope 115 amu was corrected by isotope 118 amu of $\mathrm{Sn}$ (equations are not shown).

To ensure the same operating conditions, both an external calibration series and internal standard [Ge (72 amu), Sc (45 amu), Rh (103 amu), In (115 amu) or Ir (193 amu)] were used for each sample sequence. The internal standard for each metal was chosen in relation to closed mass and ionization potential. The calibration series was prepared with the same acids as were used for samples. Calibration curves were obtained using at least five points. Some of the samples had to be diluted since they fell outside the range of the calibration. The quantification limits (LOQ) for each element are shown in Table 1, with the exception of $\mathrm{Bi}, \mathrm{Gd}$ and $\mathrm{Nd}$ : 0.1 $\mu \mathrm{g} / \mathrm{L}, 0.1 \mu \mathrm{g} / \mathrm{L}$ and $0.5 \mu \mathrm{g} / \mathrm{L}$ respectively.

\subsubsection{Quality control}


Analytical blanks and National Institute of Standards and Technology NIST 1643 were used for water analysis quality control. These control samples were inserted into all analysis series, and elements were determined in the same manner as for samples. No contamination of Sb by flask was observed. The laboratory has had French accreditation (Comité Français d'accréditation (COFRAC), 2013) for the 29 elements investigated in this study since 2007, and has participated in 2 or 3 inter-laboratory water tests per year.

\section{Results}

The results presented in Table 1 are population-based (i.e. after the sampling plan and statistical adjustment have been taken into account) and representative of the tap water of homes occupied by the 3,581,991 children aged 6 months to 6 years in France.

For some elements, such as $\mathrm{Ca}, \mathrm{Cu}, \mathrm{K}, \mathrm{Mg}, \mathrm{Mo}, \mathrm{Na}, \mathrm{Sr}, \mathrm{Zn}$ and $\mathrm{V}$, concentrations ranged across several orders of magnitude $\left(10^{3}\right.$ to $\left.10^{4}\right)$. Others, such as $\mathrm{B}, \mathrm{Cd}$, Co and Se, had a narrower range of variation - around one order of magnitude. Major elements above $1 \mathrm{mg} / \mathrm{L}$ were $\mathrm{Ca}, \mathrm{Na}$, $\mathrm{Mg}$, and $\mathrm{K}$ - yet $\mathrm{Cu}, \mathrm{Sr}$ and $\mathrm{Fe}$ also reached maximum level for this value. The median values were above quantification limits for only 9 elements ( $\mathrm{Ca}, \mathrm{Na}, \mathrm{Mg}, \mathrm{K}, \mathrm{Cu}, \mathrm{Zn}, \mathrm{Sr}, \mathrm{Ba}$, and $\mathrm{As}$ ). Of the 29 elements, $\mathrm{B}, \mathrm{Cr}, \mathrm{Mn}, \mathrm{Be}, \mathrm{Ce}, \mathrm{Tl}, \mathrm{Cd}$, and $\mathrm{Sb}$ had concentrations above LOQ in less than $6 \%$ of samples (Fig.2), whereas Bi, Gd, Nd concentrations were always below the quantification limits. 
Table 1. Tap water concentrations with confidence intervals (CI95\%) in French homes inhabited by children (2008-2009).

\begin{tabular}{|c|c|c|c|c|c|c|c|c|c|c|c|c|c|c|c|}
\hline & $\mathbf{n}$ & $\mathbf{N}$ & LOQ & Unit & AM & SD & GM & GSD & $\min$ & P5 & P25 & P50 & P75 & P95 & $\max$ \\
\hline B & 472 & 3461328 & 0.1 & $\mathrm{mg} / \mathrm{L}$ & 0.1 & $<0.1$ & 0.1 & 1.3 & $<0.1$ & $<0.1$ & $<0.1$ & $<0.1$ & $<0.1$ & $0.1(<0.2-0.3)$ & 0.3 \\
\hline $\mathrm{Ca}$ & 470 & 3459480 & 1 & $\mathrm{mg} / \mathrm{L}$ & 76 & 60.7 & 57.6 & 2.6 & $<1$ & $7.9(1.5-17)$ & $45.5(34.7-57.6)$ & $85(69.4-88.6)$ & $100.9(94.6-104)$ & 121.7 (112.7-133.5) & 1,150 \\
\hline $\mathrm{Cu}$ & 472 & 3461328 & 0.02 & $\mathrm{mg} / \mathrm{L}$ & 0.15 & 0.26 & 0.07 & 3.21 & $<0.02$ & $<0.02$ & $0.03(0.02-0.03)$ & $0.07(0.06-0.08)$ & $0.14(0.11-0.2)$ & $0.72(0.41-1.22)$ & 5.2 \\
\hline K & 472 & 3461328 & 0.05 & $\mathrm{mg} / \mathrm{L}$ & 2.64 & 2.02 & 2.01 & 2.19 & $<0.05$ & $0.43(0.29-0.59)$ & $1.17(1.05-1.43)$ & $2.21(1.78-2.58)$ & $3.37(3.05-3.58)$ & $6.74(4.3-14.3)$ & 14.3 \\
\hline Mg & 472 & 3461328 & 0.5 & $\mathrm{mg} / \mathrm{L}$ & 8.2 & 6.2 & 5.9 & 2.6 & $<0.5$ & $1.1(<0.5-2.1)$ & $3.9(3.5-4.4)$ & $6.5(5.4-7.2)$ & $11(9.3-13.3)$ & $21.2(17-37.6)$ & 37.6 \\
\hline $\mathrm{Na}$ & 467 & 3395741 & 2 & $\mathrm{mg} / \mathrm{L}$ & 22.3 & 33.5 & 14.1 & 2.4 & $<2$ & $3.3(<2-4.2)$ & $9.8(7.9-11.2)$ & $14.5(13-16.5)$ & $19(17.7-22.4)$ & $66.8(49.3-199.1)$ & 216 \\
\hline $\mathrm{Zn}$ & 458 & 3357666 & 0.005 & $\mathrm{mg} / \mathrm{L}$ & 0.081 & 0.09 & 0.057 & 2.21 & $<0.005$ & $0.016(0.015-0.017)$ & $0.029(0.027-0.032)$ & $0.053(0.044-0.062)$ & $0.087(0.076-0.109)$ & $0.208(0.167-0.411)$ & 0.86 \\
\hline Al & 471 & 3457083 & 10 & $\mu \mathrm{g} / \mathrm{L}$ & 16.3 & 31.4 & $<10$ & $<10$ & $<10$ & $<10$ & $<10$ & $<10$ & $15.4(11.9-23.5)$ & $48.3(42.2-72.6)$ & 359 \\
\hline As & 472 & 3461328 & 0.2 & $\mu g / L$ & 0.5 & 0.8 & 0.3 & 2.8 & $<0.2$ & $<0.2$ & $<0.2$ & $0.2(0.2-0.3)$ & $0.5(0.3-0.8)$ & $2.1(1.5-3.7)$ & 8.9 \\
\hline $\mathrm{Ba}$ & 472 & 3461328 & 4 & $\mu \mathrm{g} / \mathrm{L}$ & 43.9 & 59.5 & 19.4 & 4.5 & $<4$ & $<4$ & $<4$ & $30.7(27.1-35.1)$ & $50.1(41.1-67.7)$ & $149.4(101.3-230.5)$ & 703 \\
\hline $\mathrm{Be}$ & 466 & 3411328 & 0.5 & $\mu \mathrm{g} / \mathrm{L}$ & $<0.5$ & $<0.5$ & $<0.5$ & 1 & $<0.5$ & $<0.5$ & $<0.5$ & $<0.5$ & $<0.5$ & $<0.5$ & 0.7 \\
\hline Cd & 472 & 3461328 & 0.5 & $\mu \mathrm{g} / \mathrm{L}$ & $<0.5$ & $<0.5$ & $<0.5$ & 1.3 & $<0.5$ & $<0.5$ & $<0.5$ & $<0.5$ & $<0.5$ & $<0.5$ & 2.3 \\
\hline $\mathrm{Ce}$ & 472 & 3461328 & 0.5 & $\mu g / L$ & $<0.5$ & $<0.5$ & $<0.5$ & 1 & $<0.5$ & $<0.5$ & $<0.5$ & $<0.5$ & $<0.5$ & $<0.5$ & 0.5 \\
\hline Co & 472 & 3461328 & 0.5 & $\mu \mathrm{g} / \mathrm{L}$ & $<0.5$ & $<0.5$ & $<0.5$ & 1.4 & $<0.5$ & $<0.5$ & $<0.5$ & $<0.5$ & $<0.5$ & $0.8(<0.5-1.2)$ & 2.3 \\
\hline $\mathrm{Cr}$ & 472 & 3461328 & 5 & $\mu g / L$ & $<5$ & $<5$ & $<5$ & $<5$ & $<5$ & $<5$ & $<5$ & $<5$ & $<5$ & $<5$ & 17 \\
\hline $\mathrm{Fe}$ & 472 & 3461328 & 20 & $\mu \mathrm{g} / \mathrm{L}$ & 26 & 132 & $<20$ & $<20$ & $<20$ & $<20$ & $<20$ & $<20$ & $<20$ & $46(34-96)$ & 2 \\
\hline Mn & 472 & 3461328 & 5 & $\mu g / L$ & $<5$ & 5.1 & $<5$ & $<5$ & $<5$ & $<5$ & $<5$ & $<5$ & $<5$ & $<5$ & 341 \\
\hline Mo & 470 & 3457653 & 0.5 & $\mu g / L$ & 0.5 & 0.8 & $<0.5$ & 1.9 & $<0.5$ & $<0.5$ & $<0.5$ & $<0.5$ & $<0.5$ & $1.5(1.1-2.6)$ & 23 \\
\hline $\mathrm{Ni}$ & 417 & 3115321 & 2 & $\mu \mathrm{g} / \mathrm{L}$ & 2.5 & 3.9 & $<2$ & 2.2 & $<2$ & $<2$ & $<2$ & $<2$ & $2(<2-3.6)$ & $10.2(4.2-84)$ & 84 \\
\hline $\mathrm{Pb}$ & 472 & 3461328 & 1 & $\mu \mathrm{g} / \mathrm{L}$ & 1.8 & 4 & $<1$ & 2.5 & $<1$ & $<1$ & $<1$ & $<1$ & $1.1(<1-1)$ & $5.4(3.9-9.5)$ & 74 \\
\hline Sb & 472 & 3461328 & 0.5 & $\mu \mathrm{g} / \mathrm{L}$ & $<0.5$ & $<0.5$ & $<0.5$ & 1.4 & $<0.5$ & $<0.5$ & $<0.5$ & $<0.5$ & $<0.5$ & $<0.5$ & 7.4 \\
\hline Se & 469 & 3457285 & 1 & $\mu \mathrm{g} / \mathrm{L}$ & 1.1 & 1.7 & $<1$ & 2.1 & $<1$ & $<1$ & $<1$ & $<1$ & $<1$ & $\begin{array}{l}6.7(1.3-9.9) \\
1,004.6 \quad \text { (963.8- }\end{array}$ & 9.9 \\
\hline $\mathrm{Sr}$ & 472 & 3461328 & 0.5 & $\mu \mathrm{g} / \mathrm{L}$ & 380 & 498.9 & 226.1 & 3.2 & $<0.5$ & $23.4(4.5-65.7)$ & $124.5(98.6-153.3)$ & $256.9(209-309)$ & $493(358.5-644.9)$ & 1525.2) & 17,300 \\
\hline TI & 472 & 3461328 & 0.5 & $\mu \mathrm{g} / \mathrm{L}$ & $<0.5$ & $<0.5$ & $<0.5$ & 1 & $<0.5$ & $<0.5$ & $<0.5$ & $<0.5$ & $<0.5$ & $<0.5$ & 0.7 \\
\hline U & 472 & 3461328 & 0.5 & $\mu \mathrm{g} / \mathrm{L}$ & 0.7 & 1.1 & 0.5 & 2.2 & $<0.5$ & $<0.5$ & $<0.5$ & $<0.5$ & $0.7(0.6-0.9)$ & $2.4(1.4-3.4)$ & 10.1 \\
\hline v & 472 & 3461328 & 1 & $\mu \mathrm{g} / \mathrm{L}$ & 5.3 & 61.1 & $<1$ & 2 & $<1$ & $<1$ & $<1$ & $<1$ & $1(<1-1)$ & $1(1-1)$ & 816 \\
\hline
\end{tabular}

$\mathrm{n}$ : number of samples with validated result; $\mathrm{N}$ : number of represented dwellings in the population; LOQ: quantification limit; AM: arithmetic mean; SD: standard deviation; GM: geometric mean; GSD: geometric standard deviation; P:percentile 


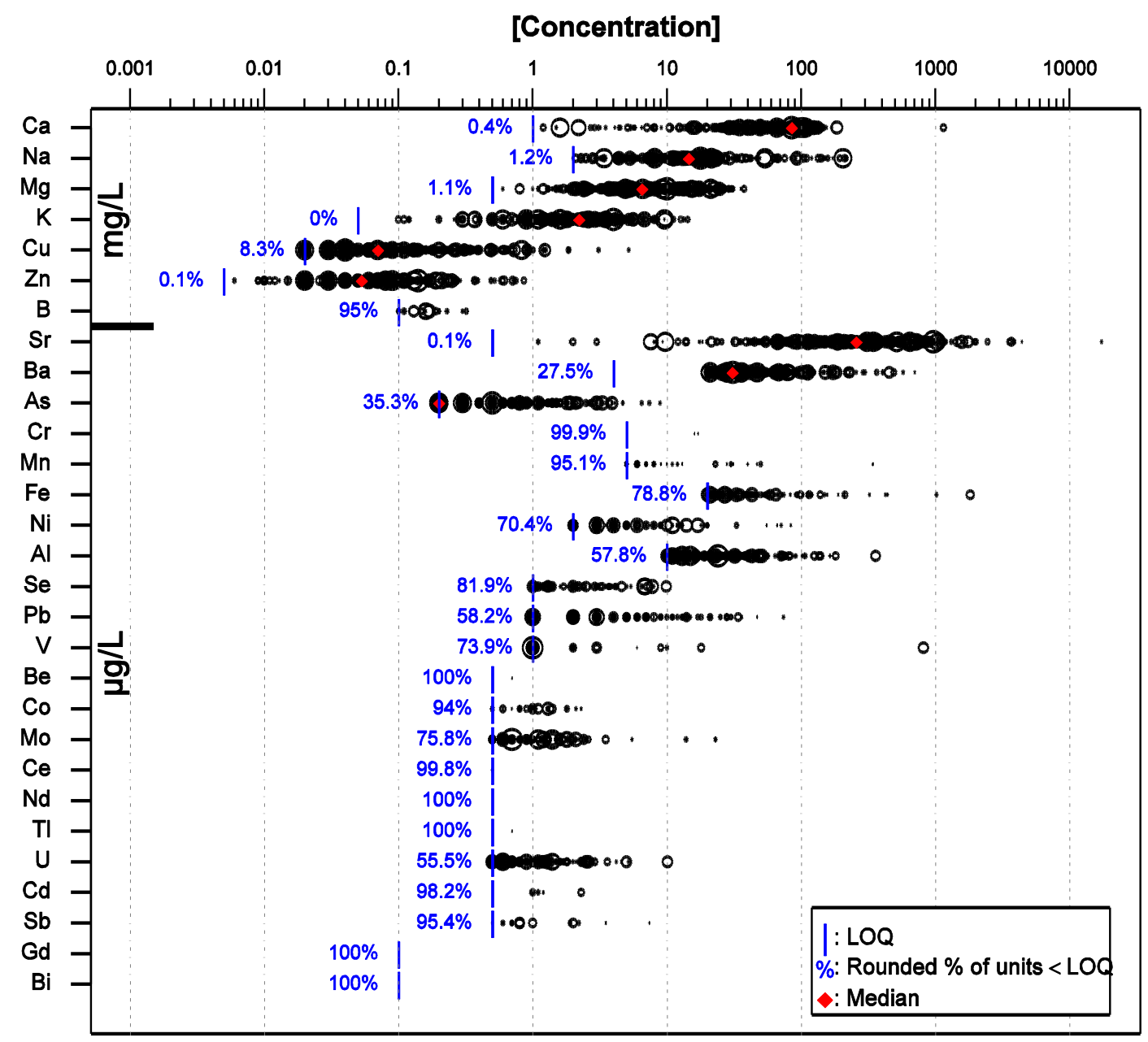

Fig. 2 Concentration of 29 elements in tap water at the homes of children, France 2008-2009.

Each bubble represents a home inhabited by children, the radius of which is proportional to the number of homes inhabited by children represented by this home in the population based. The graph includes percentages of samples under quantification limit and median concentration for each element. Elements are classified according to units and in decreasing median order. 
Comparison with recommended maximum concentrations by health authorities is shown in Table 2. These maximum concentrations have been set for 19 of the elements investigated ( $\mathrm{Al}, \mathrm{As}, \mathrm{B}$, $\mathrm{Ba}, \mathrm{Be}, \mathrm{Cd}, \mathrm{Cr}, \mathrm{Cu}, \mathrm{Fe}, \mathrm{Mn}, \mathrm{Mo}, \mathrm{Na}, \mathrm{Ni}, \mathrm{Pb}, \mathrm{Sb}, \mathrm{Se}, \mathrm{Tl}, \mathrm{U}$, and $\mathrm{Zn}$ ) by such regulatory authorities as the European Union (European Directive 1998/83EC, 1998), WHO (World Health Organization, 2011) (drinking-water guidelines) or United States Environmental Protection Agency (US EPA, 2012). In our study, As, B, Be, Cd, Cr, TI, U and Zn concentrations never exceeded any guideline or directive values. 9 elements ( $\mathrm{Al}, \mathrm{Ba}, \mathrm{Cu}, \mathrm{Fe}, \mathrm{Mn}, \mathrm{Na}, \mathrm{Ni}, \mathrm{Pb}, \mathrm{Sb}$ ) reached or exceeded one of the 3 regulatory guideline values. $\mathrm{Al}, \mathrm{Fe}, \mathrm{Mn}$ and $\mathrm{Na}$ are water quality indicator parameters (European Directive 1998/83EC, 1998) or secondary parameters having no direct impact on human health (World Health Organization, 2011), (US EPA, 2012). Only $\mathrm{Pb}, \mathrm{Ni}, \mathrm{Cu}, \mathrm{Sb}$ and $\mathrm{Ba}$ reached or exceeded European Directive (European Directive 1998/83EC, 1998) or United States Environmental Protection Agency (US EPA, 2012) quality limits with potential health impact (World Health Organization, 2011). 10 elements (Bi, $\mathrm{Ca}, \mathrm{Ce}$, $\mathrm{Co}, \mathrm{Gd}, \mathrm{K}, \mathrm{Mg}, \mathrm{Nd}, \mathrm{Sr}$, and $\mathrm{V}$ ) have no reference values.

$2,977,123$ out of $4,923,058$ children (about $60 \%$ ) drank tap water, and thus were exposed to the tap water concentrations shown in Table 3. Tap water consumers were exposed to 4 elements $(\mathrm{Ba}, \mathrm{Ni}, \mathrm{Pb}, \mathrm{Sb})$ at levels in excess of the lowest guideline or concentration limit concentration (European Directive 1998/83EC, 1998), (World Health Organization, 2011), (US EPA, 2012). 78,466 (CI95\%: 17,171-139,761) children were consuming water with levels in excess of $10 \mu \mathrm{g} / \mathrm{L}$ of $\mathrm{Pb}, 7,242$ (Cl95\%: 0-18,043) children were consuming water with $\mathrm{Ni}$ concentration in excess of $20 \mu \mathrm{g} / \mathrm{L}, 719$ (CI95\%: 0-2,139) were consuming water with levels in excess of $5 \mu \mathrm{g} / \mathrm{L}$ of Sb and 498 (Cl95\%: 0-1,484) were drinking-water with levels in excess of $700 \mu \mathrm{g} / \mathrm{L}$ of Ba. 
Table 2. European Directive, World Health Organization guidelines and U.S.EPA limits for concentrations in drinking-water, and corresponding compliance of tap water supplied to homes inhabited by children, France 2008-2009.

\begin{tabular}{|c|c|c|c|c|}
\hline Elements & Units & Concentration Limits $(1,2,3)$ & $\%$ Home's tap water above (CI95\%) & $\begin{array}{l}\text { No. of Children exposed above } \\
\text { (Cl95\%) }\end{array}$ \\
\hline Al & $\mu \mathrm{g} / \mathrm{L}$ & $200(1 a)$ & $0.5(0-1.5)$ & $27,097(0-80,315)$ \\
\hline \multirow[t]{2}{*}{$\mathrm{Ba}$} & \multirow{2}{*}{$\mu \mathrm{g} / \mathrm{L}$} & $700(1,2)$ & 0 & $498(0-1,484)$ \\
\hline & & $2,000(3)$ & 0 & 0 \\
\hline \multirow[t]{2}{*}{$\mathrm{Cu}$} & \multirow{2}{*}{$\mathrm{mg} / \mathrm{L}$} & $2(1)$ & $0.1(0-0.3)$ & 0 \\
\hline & & $1.3(3)$ & $0.3(0-0.06)$ & 0 \\
\hline \multirow{2}{*}{$\mathrm{Fe}$} & \multirow{2}{*}{$\mu \mathrm{g} / \mathrm{L}$} & $200(1 a)$ & $0.9(0-2)$ & $44,189(0-102,823)$ \\
\hline & & $300(3 b)$ & $0.6(0-1.7)$ & $33,359(0-87,246)$ \\
\hline Mn & $\mu \mathrm{g} / \mathrm{L}$ & $50(1 a)$ & $0.3(0-0.9)$ & $15,388(0-44,074)$ \\
\hline \multirow{2}{*}{$\mathrm{Na}$} & \multirow[t]{2}{*}{$\mathrm{mg} / \mathrm{L}$} & $200(1 a)$ & $2.1(0-5.6)$ & $7,652(0-20,832)$ \\
\hline & & $50(2)$ & $8.7(0-17.7)$ & $121,581(7,091-236,070)$ \\
\hline \multirow[t]{2}{*}{$\mathrm{Ni}$} & \multirow[t]{2}{*}{$\mu \mathrm{g} / \mathrm{L}$} & $20(1)$ & $0.4(0-0.8)$ & $7,242(0-18,043)$ \\
\hline & & $70(2)$ & $0.1(0-0.1)$ & $2,044(0-6,132)$ \\
\hline \multirow{2}{*}{$\mathrm{Pb}$} & \multirow{2}{*}{$\mu \mathrm{g} / \mathrm{L}$} & $10(1,2)$ & $2.9(1.2-4.5)$ & $78,466(17,171-139,761)$ \\
\hline & & $15(3)$ & $1.6(0.4-2.7)$ & $51,261(0-110,844)$ \\
\hline \multirow[t]{2}{*}{ Sb } & \multirow{2}{*}{$\mu \mathrm{g} / \mathrm{L}$} & $5(1)$ & 0 & $719(0-2,139)$ \\
\hline & & $6(3)$ & 0 & $719(0-2,139)$ \\
\hline
\end{tabular}

CI 95\%: confidence interval; (1): EU directive 98/83EC concentration limits; (a): indicator parameter; (2): World Health Organization Guideline 2011; (3): United States of America Environmental Protection Agency (U.S. EPA 2012)

Maximum contaminant level; (b): secondary Maximum Contaminant level (U.S. EPA). 
Table 3. Concentrations in tap water consumed by children in France (2008-2009).

\begin{tabular}{|c|c|c|c|c|c|c|c|c|c|c|c|c|c|c|c|}
\hline & $\mathbf{n}$ & $N$ & LOQ & Unit & AM & SD & GM & GSD & $\min$ & P5 & P25 & P50 & P75 & P95 & $\max$ \\
\hline B & 270 & 2418519 & 0.1 & $\mathrm{mg} / \mathrm{L}$ & 0.1 & $<0.1$ & 0.1 & 1.1 & $<0.1$ & $<0.1$ & $\begin{array}{l}<0.1 \\
40.2\end{array}$ & $\begin{array}{l}<0.1 \\
82.9\end{array}$ & $\begin{array}{l}<0.1 \\
98.3\end{array}$ & $\begin{array}{l}<0.1 \\
120.8\end{array}$ & 0.2 \\
\hline $\mathrm{Ca}$ & 269 & 2349880 & 1 & $\mathrm{mg} / \mathrm{L}$ & 71.1 & 33.6 & 57.5 & 2.3 & $<1$ & $15.2(1.7-23.1)$ & $\begin{array}{c}(31.7-54.6) \\
0.04\end{array}$ & $\begin{array}{c}(60.9-87.1) \\
0.07\end{array}$ & $\begin{array}{c}(91-102.5) \\
0.14\end{array}$ & $\begin{array}{c}(107.8-134.3) \\
0.72\end{array}$ & 139 \\
\hline $\mathrm{K}$ & 270 & 2418519 & 0.05 & $\mathrm{mg} / \mathrm{L}$ & 2.41 & 1.55 & 1.92 & 2.09 & $<0.05$ & $\begin{array}{c}(<0.05-0.62) \\
2.7\end{array}$ & $\begin{array}{c}(1-1.49) \\
8.1\end{array}$ & $\begin{array}{c}(1.73-2.55) \\
13.7\end{array}$ & $\begin{array}{c}(2.84-3.52) \\
18\end{array}$ & $\begin{array}{c}(4.18-7.97) \\
27.1\end{array}$ & 14.3 \\
\hline $\mathrm{Na}$ & 266 & 2369353 & 2 & $\mathrm{mg} / \mathrm{L}$ & 16.8 & 20 & 12 & 2.3 & $<2$ & $\begin{array}{c}(<2-4.3) \\
1.6\end{array}$ & $\begin{array}{c}(6.5-10.8) \\
3.8\end{array}$ & $\begin{array}{c}(11.4-15.8) \\
6.1\end{array}$ & $\begin{array}{c}(16.1-20.7) \\
10\end{array}$ & $\begin{array}{c}(22.7-101.6) \\
16.5\end{array}$ & 216 \\
\hline $\mathrm{Zn}$ & 258 & 2317786 & 0.005 & $\mathrm{mg} / \mathrm{L}$ & 0.08 & 0.078 & 0.058 & 2.227 & $<0.005$ & $(0.016-0.017)$ & $(0.026-0.033)$ & $(0.038-0.072)$ & $\begin{array}{c}(0.079-0.136) \\
15.2\end{array}$ & $\begin{array}{c}(0.164-0.72) \\
49.2\end{array}$ & 0.72 \\
\hline Al & 269 & 2411958 & 10 & $\mu \mathrm{g} / \mathrm{L}$ & 17 & 38.1 & $<10$ & $<10$ & $<10$ & $<10$ & $<10$ & $\begin{array}{l}<10 \\
0.2\end{array}$ & $\begin{array}{c}(10.1-23.4) \\
0.5\end{array}$ & $\begin{array}{c}(36.9-110) \\
2.8\end{array}$ & 359 \\
\hline As & 270 & 2418519 & 0.2 & $\mu \mathrm{g} / \mathrm{L}$ & 0.6 & 0.8 & 0.3 & 3 & $<0.2$ & $<0.2$ & $<0.2$ & $\begin{array}{c}(<0.2-0.3) \\
30.6\end{array}$ & $\begin{array}{c}(0.3-1) \\
52\end{array}$ & $\begin{array}{c}(1.3-7.4) \\
166.4\end{array}$ & 7.4 \\
\hline $\mathrm{Ba}$ & 270 & 2418519 & 4 & $\mu \mathrm{g} / \mathrm{L}$ & 42.6 & 53.5 & 19.5 & 4.4 & $<4$ & $<4$ & $<4$ & $(25.3-37)$ & $(41.8-69.3)$ & $(78.9-703)$ & 703 \\
\hline Co & 270 & 2418519 & 0.5 & $\mu \mathrm{g} / \mathrm{L}$ & $<0.5$ & $<0.5$ & $<0.5$ & 1.5 & $<0.5$ & $<0.5$ & $<0.5$ & $<0.5$ & $<0.5$ & $1(<0.5-2.1)$ & 2.1 \\
\hline $\mathrm{Cr}$ & 270 & 2418519 & 5 & $\mu \mathrm{g} / \mathrm{L}$ & $<5$ & $<5$ & $<5$ & $<5$ & $<5$ & $<5$ & $<5$ & $<5$ & $<5$ & $<5$ & 16 \\
\hline $\mathrm{Fe}$ & 270 & 2418519 & 20 & $\mu \mathrm{g} / \mathrm{L}$ & 35 & 176 & $<20$ & $<20$ & $<20$ & $<20$ & $<20$ & $<20$ & $<20$ & $63(42-1507)$ & 1820 \\
\hline Gd & 270 & 2418519 & 0.1 & $\mu \mathrm{g} / \mathrm{L}$ & $<0.1$ & $<0.1$ & 0.1 & 1 & $<0.1$ & $<0.1$ & $<0.1$ & $<0.1$ & $<0.1$ & $<0.1$ & $<0.1$ \\
\hline Mo & 269 & 2417076 & 0.5 & $\mu \mathrm{g} / \mathrm{L}$ & $<0.5$ & 0.6 & $<0.5$ & 1.8 & $<0.5$ & $<0.5$ & $<0.5$ & $<0.5$ & $<0.5$ & $1.2(0.7-13.9)$ & 13.9 \\
\hline Mn & 270 & 2418519 & 5 & $\mu \mathrm{g} / \mathrm{L}$ & $<5$ & 7.1 & $<5$ & $<5$ & $<5$ & $<5$ & $<5$ & $<5$ & $<5$ & $5(<5-16.9)$ & 341 \\
\hline Nd & 266 & 2382886 & 0.5 & $\mu g / L$ & $<0.5$ & $<0.5$ & $<0.5$ & 1 & $<0.5$ & $<0.5$ & $<0.5$ & $<0.5$ & $<0.5$ & $<0.5$ & $<0.5$ \\
\hline $\mathrm{Ni}$ & 240 & 2093678 & 2 & $\mu g / L$ & 2.4 & 3.7 & $<2$ & 2.2 & $<2$ & $<2$ & $<2$ & $<2$ & $2(<2-3.7)$ & $7.9(4.4-71)$ & 71 \\
\hline $\mathrm{Pb}$ & 270 & 2418519 & 1 & $\mu \mathrm{g} / \mathrm{L}$ & 1.9 & 4.3 & $<1$ & 2.5 & $<1$ & $<1$ & $<1$ & $<1$ & $2(1-2)$ & $7(4-11)$ & 47 \\
\hline Sb & 270 & 2418519 & 0.5 & $\mu \mathrm{g} / \mathrm{L}$ & $<0.5$ & $<0.5$ & $<0.5$ & 1.4 & $<0.5$ & $<0.5$ & $<0.5$ & $<0.5$ & $<0.5$ & $<0.5$ & 7.4 \\
\hline Se & 268 & 2398373 & 1 & $\mu g / L$ & $<1$ & 1.3 & $<1$ & 1.8 & $<1$ & $\begin{array}{l}<1 \\
51.4\end{array}$ & $\begin{array}{l}<1 \\
112.4\end{array}$ & $\begin{array}{c}<1 \\
244.5\end{array}$ & $\begin{array}{c}<1 \\
514.8\end{array}$ & $\begin{array}{c}3(1.2-9.9) \\
996.9\end{array}$ & 9.9 \\
\hline
\end{tabular}

$\mathrm{n}$ : number of samples with validated result; N: number of children; LOQ: quantification limit; AM: arithmetic mean; SD:

standard deviation; GM: geometric mean; GSD: geometric standard deviation; P:percentile; number in brackets are $95 \%$ confidence intervals. 


\section{Discussion}

This is the first study to provide representative estimations of inorganic element concentrations in the tap water of homes inhabited by children in France.

\subsection{Origins of elements exceeding guidelines}

The 9 elements ( $\mathrm{Al}, \mathrm{Ba}, \mathrm{Cu}, \mathrm{Fe}, \mathrm{Mn}, \mathrm{Na}, \mathrm{Ni}, \mathrm{Pb}$, and $\mathrm{Sb}$ ) for which concentrations exceed one of the maximum levels recommended in drinking-water by regulatory authorities worldwide (Table 2) have different origins and uses that may explain their presence in tap water. Aluminium, sodium, iron and manganese are elements that are abundant in the earth's crust; barium and antimony are metals occurring in natural ores, and so are naturally present in water. Lead, copper, nickel and iron are metals used to make pipes, taps or fittings in water distribution systems, and can thus be released in tap water depending on several factors - including $\mathrm{pH}$, temperature, water hardness and standing time of the water in pipes, as well as whether the water is soft or acidic (Veschetti et al., 2010). Some elements, such as aluminium and iron salts, are also used in water treatment plants as coagulants, and copper sulphate pentahydrate is added to surface water to control algae (Dinelli et al., 2012). Barium can be naturally present in the environment, or discharged during ore extraction, refinement or processing in manufacturing activities (Host S. et al., 2005). Sb was also naturally present in water and is used in many manufacturing activities - though $\mathrm{Sb}$ has also been shown to be released from polyethylene containers.

\subsection{Comparison with other studies}

As shown in Table 2, $\mathrm{Pb}, \mathrm{Ni}$ and $\mathrm{Cu}$ had the highest percentages of concentrations in excess of the quality limits of regulatory authorities worldwide (European Directive 1998/83EC, 1998), (World Health Organization, 2011) or (US EPA, 2012) and were also known to be chemical constituents released from distribution system materials (Veschetti et al., 2010) (Volker et al., 2010). French health authorities monitor drinking-water quality in order to comply with drinkingwater regulation for human consumption (European Directive 1998/83EC, 1998). B, Ba, Cd, Cr, 
$\mathrm{Cu}, \mathrm{Fe}, \mathrm{Ni}, \mathrm{Pb}, \mathrm{Sb}$ and $\mathrm{Se}$ concentrations are available for drinking-water quality produced in 2006 (Davezac H. et al., 2008). Between 15,000 and 18,000 tap water samples were analysed in all of France for all elements and 34,300 for $\mathrm{Pb}$, excluding complementary and rechecking analysis. These samples were selected randomly but in accordance with the size of population using the water network. The comparison of results is tricky because the sample representativeness and sample methods are not the same). It nevertheless showed that the same elements $(\mathrm{Cu}, \mathrm{Ni}$ and $\mathrm{Pb})$ exceeded quality limits. Regulatory monitoring observed that $\mathrm{Ba}$, B, Cd, Cr, Sb and Se were also non-compliant in 2006, (Davezac H. et al., 2008) although these were not observed in our study. Differences could be explained by sampling point on the distribution network, sampling method and public concerned (only children aged up to 6 years, in our study). In this study, all tap water was sampled in the kitchens of homes inhabited by children - the place where children most often drank water. Sampling took the form of a 30minute stagnation sample, after a cold water flush and 30 minutes standing, in order to limit metals solubilisation from taps and pipes to water (De Brouwere et al., 2012;Hoekstra E.J. et al., 2004; Veschetti et al., 2010). The water safety regulatory monitoring sampling method omitted the pre-sampling flush (Legifrance, 2003). Veschetti and al. have observed the difference in metals concentrations when taps are flushed prior to sampling (Veschetti et al., 2010).

At the European level, 579 tap water sample concentrations are available from the EuroGeoSurveys study, which investigated 60 parameters, including metals and metalloids, in 2009 (Banks D., et al. 2015). These samples were collected at end-consumer taps, run for 5 minutes, using empty and thoroughly-rinsed mineral water bottles without acidification. Twelve samples came from France. No instruction was given regarding sampling representativeness of neither population nor ultimate potable water source, and thus results comparability with our results is limited. It nevertheless offers a large and unique data set to compare at the European level, as done in the following lines. Among the 579 samples, $0.5 \%$ of $\mathrm{Pb}$ concentrations were 
above the current tolerable concentration laid down by European water legislation $(10 \mu \mathrm{g} / \mathrm{L})$, as were $0.2 \%$ of $\mathrm{Ni}$ concentrations $(20 \mu \mathrm{g} / \mathrm{L})$. No copper concentration exceeded the $2 \mathrm{mg} / \mathrm{L}$ reference value. These results are very similar to ours. The percentile 75 of Sb concentration observed in tap water in France was below $0.5 \mu \mathrm{g} / \mathrm{L}$ (Table 1), as in tap water from the European survey. Maximum Ba concentration observed in French tap water reached the maximum EU Directive concentration level $(700 \mu \mathrm{g} / \mathrm{L})$, whereas the European study's tap water reached $1660 \mu \mathrm{g} / \mathrm{L}$. Among the 10 elements for which no reference values exist, seven had concentrations above quantification limits ( $\mathrm{Ca}, \mathrm{Ce}, \mathrm{Co}, \mathrm{K}, \mathrm{Mg}, \mathrm{Sr}, \mathrm{V})$. Calcium and magnesium form the major common elements in water and are abundant in the earth's crust. Some European countries had formerly established calcium limits for practical reasons (to protect water systems against limescale). Calcium had the highest median concentration ( $85 \mathrm{mg} / \mathrm{L})$, with broad variability. In the European survey similar results were observed (median concentration 60 $\mathrm{mg} / \mathrm{L} ; \mathrm{n}=579$ tap water samples). Magnesium median concentration was lower (6.5 mg/L) and similar to European tap water samples $(9.6 \mathrm{mg} / \mathrm{L} ; \mathrm{n}=579)$. Cerium is a heavy metal and the most abundant of rare earth elements. Occurrence of $\mathrm{Ce}$ in groundwater is linked to geological origin. In our study, P99 is the lower than quantification limit $(0.5 \mu \mathrm{g} / \mathrm{L})$ as in $98 \%$ of tap water samples of the European survey. Sources of environmental cobalt are both natural and anthropogenic (Barceloux, 1999). Cobalt is a heavy metal present in many common minerals and used industrial products. But Co is also a micronutrient essential for human health. There is no internationally-defined maximum level for Co in water, but in the European Union, cobalt chloride and sulphate are classified as being possibly carcinogenic to humans (Cobalt sulphate and other soluble cobalt(II) salts; Group 2B) (WHO: 2006). P75 concentration in French homes is the LOQ of $0.5 \mu \mathrm{g} / \mathrm{L}$ consistent with values observed in European tap water $(P 75=0.05 \mu \mathrm{g} / \mathrm{L} / \mathrm{n}=579)$. Potassium is abundant in the earth's crust, and necessary for the functioning of all living cells. The median concentration observed $(2.2 \mathrm{mg} / \mathrm{L})$ is similar to that observed in European tap water $(1.6 \mathrm{mg} / \mathrm{L})$. $\mathrm{Sr}$ is an alkaline metal abundant on the earth in mineral forms $\left(\mathrm{SrSO}_{4}\right.$ or $\left.\mathrm{SrCO}_{3}\right)$. 
Strontium is usually mobilized during weathering, and accumulated in natural waters. The median concentration observed in tap water consumed by children in France $(257 \mu \mathrm{g} / \mathrm{L}$, IC95\%:209-309) is slightly higher than median concentration observed in European tap water (P50=177 $\mu \mathrm{g} / \mathrm{L} ; \mathrm{n}=579$ ) but with the same wide variability (4 to 5 orders of magnitude). Vanadium is a metallic constituent of many minerals and has a significant presence in crude oil. Vanadium is used in producing rust-resistant, spring, and high-speed tool steels (ATSDR (Agency for toxic substances and diseases registry), 2012). The P75 concentration observed in French tap water was $1 \mu \mathrm{g} / \mathrm{L}$ against $0.4 \mu \mathrm{g} / \mathrm{L}$ in European tap water survey $(\mathrm{n}=579)$.

\subsection{Compliance to guidelines}

$2,977,123$ out of $3,581,991$ young children in France were consuming home tap water complying with the World Health Organization drinking-water guidelines (WHO 2011) for As, B, Ba, Cd, Cr, $\mathrm{Cu}, \mathrm{Sb}, \mathrm{Se}$, and $\mathrm{U}$. Because of the relatively low sample size, estimates of the number of children consuming tap water non-compliant with guidelines have large confidence intervals. Actually, when estimating the number of children consuming tap water non-compliant with guidelines, most confidence intervals for include 0, except for lead. Indeed, 78,500 (IC95: $17,200-140,000)$ children were consuming water containing over $10 \mu \mathrm{g} / \mathrm{L}$ of lead. Lead in water has been confirmed to contribute to blood lead levels in children, even at low concentrations (Levallois et al. 2013; Oulhote et al. 2013; Etchevers et al. 2015). Although French data are not published for more precise age groups, we suppose water consumption may vary a lot within the 6 months to 6 years age span. Even if not addressed here, the use of tap water for cooking may also contribute to exposure (Etchevers et al. 2015) and children may also be exposed outside home, at school or kindergarten for instance. Health effects may occur even at the new drinkingwater standard for lead: the EFSA (Panel on Contaminants in the Food Chain (CONTAM) 2010) concluded that with consumption of drinking-water with lead at a concentration of $2.1 \mu \mathrm{g} / \mathrm{L}$, the dietary exposure of sensitive subgroups (infants and foetuses) to lead results in a margin-ofexposure value of less than 1 - indicating that risks to young children regarding 
neurodevelopmental effects cannot be excluded. In fact, whereas thousands of children are exposed to levels in excess of the current guideline, lowering lead in drinking-water to below the current guideline of $10 \mu \mathrm{g} / \mathrm{L}$ would be beneficial to Public Health, because a further reduction in lead intake is warranted for risk reduction (SCHER (Scientific Committee on Health and Environmental Risks) 2011)). In this perspective, the replacement of old lead water pipes would be useful.

\section{Conclusion}

This nationwide survey provided unique estimates, representative both in terms of housing and in terms of children's consumption of tap water. We estimated 29 inorganic element concentrations in the tap water of 3,581,991 homes of children aged 6 months to 6 years in metropolitan France, and evaluated contamination in tap water consumed by 2,977,123 children. These results can be used in conjunction with diet and environmental (dust and soil) exposure data in an integrated metal exposure assessment for children in France. They may be usefully completed by data on water quality in other life places.

\section{Acknowledgments}

This research was funded by the French ministries in charge of health, ecology, and housing. Funding bodies did not take part in study design, data analysis nor interpretation.

The authors thank Séverine Durand, Erwann Gilles and Claire Arcelin for help in laboratory work. For helpful comment on the manuscript and English correction, we thank Jane Roffe. 


\section{References}

Agence française de normalisation, 2004. Water quality - Sampling Part3: Guidance on the preservation and handling of water samples. Association Française de Normalisation, La Plaine Saint-Denis.

ATSDR (Agency for toxic substances and diseases registry), 2012. Toxic Substances Portal Vanadium. http://www.atsdr.cdc.gov/toxfaqs/TF.asp?id=275\&tid=50.

Barceloux D.G., 1999. Cobalt. Journal of Toxicology - Clinical Toxicology; 37: 201-216.

Comité Français d'accréditation (COFRAC), 2013. ISO 17025, http://www.cofrac.fr/annexes/sect1/1-1951.pdf.

Davezac H., Grandguillot G., Robin A., and Saout C., 2008. Contrôle sanitaire 2005-2006. Delegation à l'information et à la communication Ministere de la Sante, de la Jeunesse, des Sports et de la Vie associative, Paris.

De Brouwere K., Buekers J., Cornelis C., Schlekat C.E., Oller A.R., 2012. Assessment of indirect human exposure to environmental sources of nickel: Oral exposure and risk characterization for systemic effects. Science of the Total Environment 419: 25-36.

Dinelli E., Lima A., Albanese S., Birke M., Cicchella D., Giaccio L., Valera P., De Vivo B., 2012. Major and trace elements in tap water from Italy. Journal of Geochemical Exploration 112: 5475.

Etchevers A., Bretin P., Lecoffre C., Bidondo M.L., Le Strat Y., Glorennec P., Le Tertre A., 2014. Blood lead levels and risk factors in young children in France, 2008-2009. International Journal of Hygiene and Environmental Health 217: 528-537.

Etchevers, A., Le Tertre, A., Lucas, J-P., Bretin, P., Oulhote, Y., Le Bot, B., Glorennec, P. 2015. Environmental determinants of different blood lead levels in children: A quantile analysis from a nationwide survey, Environment International 74:152-159.

Giaccio L., Cicchella D., De Vivo B., Lombardi G., De Rosa M., 2012. Does heavy metals pollution affects semen quality in men? A case of study in the metropolitan area of Naples (Italy). Journal of Geochemical Exploration 112: 218-225.

Glorennec P., Lucas J.P., Mandin C., Le Bot B., 2012. French children's exposure to metals via ingestion of indoor dust, outdoor playground dust and soil: Contamination data. Environment International 45: 129-134.

Hoekstra E.J., Pedroni V., Passarella R., Trincherini P.R., and Eisenreich S.J., 2004. Elements in tap water - part 3: effect of sample volume and stagnation time on the concentration of the element. EUR 20672 EN/3. Report of the European Commission. Joint Research Centre.

Host S., Krakowiak D., and Elluin M., 2005. Rapport IGS, Atelier Santé Environnement : Evaluation et gestion de l'exposition au Baryum. 
Ibanez Y., Le Bot B., Glorennec P., 2010. House-dust metal content and bioaccessibility: A review. European Journal of Mineralogy 22: 629-637.

EFSA Panel on Contaminants in the Food Chain (CONTAM), 2010. Scientific Opinion on Lead in Food. EFSA Journal 8: 1-147.

Fantino M., Gourmet E., 2008. Nutrient intakes in 2005 by non-breast fed French children of less than 36 months. Archives de Pediatrie 15: 446-455.

Legifrance, 2003. Arrêté du 31 décembre 2003 relatif aux conditions d'échantillonnage à mettre en œuvre pour mesurer $\mathrm{Pb}, \mathrm{Cu}, \mathrm{Ni}$ dans les eaux destinées à la consommation humaine pris en application de l'article R.1321-20 du code de la santé publique 21-1-2004.

Ref Type: Bill/Resolution

Levallois P., St-Laurent J., Gauvin D., Courteau M., Prevost M., Campagna C., et al. 2013. The impact of drinking-water, indoor dust and paint on blood lead levels of children aged 1-5 years in Montreal (Quebec, Canada). J Expo Sci Environ Epidemiol.

Lioret S., Dubuisson C., Dufour A., Touvier M., Calamassi-Tran G., Maire B., Volatier J.L., Lafay L., 2010. Trends in food intake in French children from 1999 to 2007: Results from the INCA (Etude Individuelle Nationale des Consommations Alimentaires) dietary surveys. British Journal of Nutrition 103: 585-601.

Lucas J.P., Le Bot B., Glorennec P., Etchevers A., Bretin P., Douay F., Sébille V., Bellanger L., Mandin C., 2012. Lead contamination in French children's homes and environment. Environmental research 116: 58-65.

Lucas J.P.,Sébille V., Le Tertre A., Le Strat Y., Bellanger L., 2014. Multilevel modelling of survey data: impact of the two-level weights used in the pseudolikelihood, Journal of Applied Statistics 41:4, 716-732.

Lumley T., 2010a. Complex Surveys. John Wiley \& Sons. Inc.. Hoboken. NJ. USA.

Lumley T., 2010b. Survey: analysis of complex survey samples. R package version 3.22-4.

Mielke H.W., Laidlaw M.A.S., Gonzales C., 2010. Lead (Pb) legacy from vehicle traffic in eight California urbanized areas: Continuing influence of lead dust on children's health. Science of the Total Environment 408: 3965-3975.

Nahar M.S., Zhang J., 2012. Assessment of potable water quality including organic, inorganic, and trace metal concentrations. Environmental Geochemistry and Health 34: 141-150.

Oulhote Y., Tertre A.L., Etchevers A., Le Bot B., Lucas J.P., Mandin C, et al. 2013. Implications of different residential lead standards on children's blood lead levels in France: Predictions based on a national cross-sectional survey. Int J Hyg Environ Health. Nov, 216(6):743-50.

Peter A.L.J., Viraraghavan T., 2005. Thallium: a review of public health and environmental concerns. Environment International 31: 493-501.

Banks, D., Birke, M., Flem, B., \& Reimann, C. (2014). Inorganic chemical quality of European tap-water: 1. Distribution of parameters and regulatory compliance. Applied Geochemistry 59: 200-210. 
SCHER (Scientific Committee on Health andEnvironmental Risks). 2011. Opinion on Lead Standard in Drinking-water. Brussels:European commission.

Stalder E., Blanc A., Haldimann M., Dudler V., 2012. Occurrence of uranium in Swiss drinkingwater, Chemosphere 86: 672-679.

US EPA, 2012. Guidelines for drinking-water quality, Edition of the Drinking-water Standards and Health

AdvisoriesEPA

822-S-12-001

http://water.epa.gov/action/advisories/drinking/upload/dwstandards2012.pdf. Office of Water U.S. Environmental Protection Agency Washington, DC, Spring 2012, Date of update: April, 2012.

Veschetti E., Achene L., Ferretti E., Lucentini L., Citti G., Ottaviani M., 2010. Migration of trace metals in italian drinking-waters from distribution networks. Toxicological and Environmental Chemistry 92: 521-535.

Villaescusa I., Bollinger J.C.,2008 .Arsenic in drinking-water: Sources, occurrence and health effects (a review). Reviews in Environmental Science and Biotechnology 7: 307-323.

Volker S., Schreiber C., Kistemann T., 2010. Drinking-water quality in household supply infrastructure-A survey of the current situation in Germany. International Journal of Hygiene and Environmental Health 213: 204-209.

WHO.World Health Organization. 2011. Guidelines for drinking-water qualityRecommendations. Geneva:World Health Organization.

WHO, 2010. IPCS (International programme on chemical safety). Environmental Health Criteria, Strontium and strontium compounds.Geneva. http://www.who.int/ipcs/publications/cicad/cicad77.pdf.

WHO, 2006. IPCS (International programme on chemical safety). Environmental Health Criteria, Cobalt and inorganic cobalt compounds.Geneva.

Available on: http://www.who.int/ipcs/publications/cicad/cicad69\%20.pdf. n 69.89 p.

Zietz B.P., Lass J., Suchenwirth R., Dunkelberg H., 2010. Lead in Drinking-water as a Public Health Challenge. Environmental Health Perspectives 118: A154-A155. 